\title{
Optimisation of Soft X-Ray Spectroscopy at Room and Liquid Nitrogen Temperatures
}

\author{
C.M. MacRae ${ }^{1}$, N.C. Wilson ${ }^{1}$ and A. Torpy ${ }^{1}$ \\ 1. CSIRO Mineral Resources, Microbeam Laboratory, Clayton South, Australia.
}

Field emission equipped electron probe microanalysers (FEG-EPMA) offer high beam power density at a range of accelerating voltages, and excellent beam stability over days of operation. However, with high beam current densities the onset of damage occurs faster [1]. To reduce the onset of beam damage we have integrated a liquid nitrogen (LN) cold stage into a modern FEG-EPMA. This new design of LN cold stage is able to perform both stage and beam mapping, offering the advantage over normal cold stages in that large area maps up to several $\mathrm{mm}^{2}$ can be collected at cryogenic temperatures using a variety of detectors such as grating optical and x-ray spectrometers for hyperspectral soft $\mathrm{x}$-ray emission spectroscopy (SXES). We have optimised the collection strategies of our camera which employs a back-thinned Princeton CCD. This has reduced the collection noise and allows the low energy response to be optimised.

Measuring spectra at low temperature has the additional advantage of reducing the peak width due to thermal smearing and hence sharpening the Fermi edge. In the case of SXES we have examined the Al; L Fermi edge $(72.5 \mathrm{eV})$ at both room and LN temperatures (Fig. 1) and observe that the Al; L peak shifts to higher energy by up to $30 \mathrm{meV}$ at LN temperature, but the base does not move, resulting in a sharper edge. Spectra were collected on the Al specimen at $7 \mathrm{kV}, 40 \mathrm{nA}, 1.5 \mathrm{~s}$ acquisitions averaged over $200 \mathrm{~s}$. Using the $25 \%$ to $75 \%$ of the Al peak height as a measure of the energy spread we fit a line to the Fermi edge and at room temperature the energy resolution $257 \mathrm{meV}$ is observed while at $\mathrm{LN} 246 \mathrm{meV}$ is observed. The broadening seen here is consistent with the Fermi-Dirac distribution at room temperature that follows the $4 k \mathrm{~T}$ rule which for SXES has been predicted by Terauchi et al. [2] and observed by Callcott and Arakawa [3].

The noise optimisation has enhanced the SXES's ability to detect both Li; K emission in ionically bonded compounds for example LiF, Fig. 2. The LiF was mapped at $3 \mathrm{kV}, 80 \mathrm{nA}, 20$ micron spot, $5 \mathrm{~s}$ dwell. An electrically conductive Ir film, 2nm, was applied to minimise software x-ray absorption. Spectra were averaged to reduce noise and mapping was employed to minimise beam damage. We observe the $\mathrm{Li}$; $\mathrm{K}$ emission normally which is normally centred at $54 \mathrm{eV}$ in metals, is split into two peaks centred at $44.8 \mathrm{eV}$ and $60.6 \mathrm{eV}$. This is in agreement with soft $\mathrm{x}$-ray emission spectra measured using a reflection zone plate wavelength dispersive spectrometer fitted to a soft x-ray end station at Bessy II KMC-2 beamline [4], where they report $\mathrm{Li}$; $\mathrm{K}$ peaks at $46.5 \mathrm{eV}$ and $61 \mathrm{eV}$ in $\mathrm{LiF}$ [5].

\section{References:}

[1] C.M. MacRae, N.C., Wilson and A. Torpy, Mineralogy and Petrology 107 (2013), p 429.

[2] M. Terauchi and M. Kawana, Ultramicroscopy 106 (2006), p. 1069.

[3] T.A. Callcott and E.T. Arakawa, Physical Review Letters, 38 (1977), p 442.

[4] Hafner et al, Optics Express 23 (2015), p. 29476.

[5] The authors acknowledge the support of the ARC, LE130100087. 


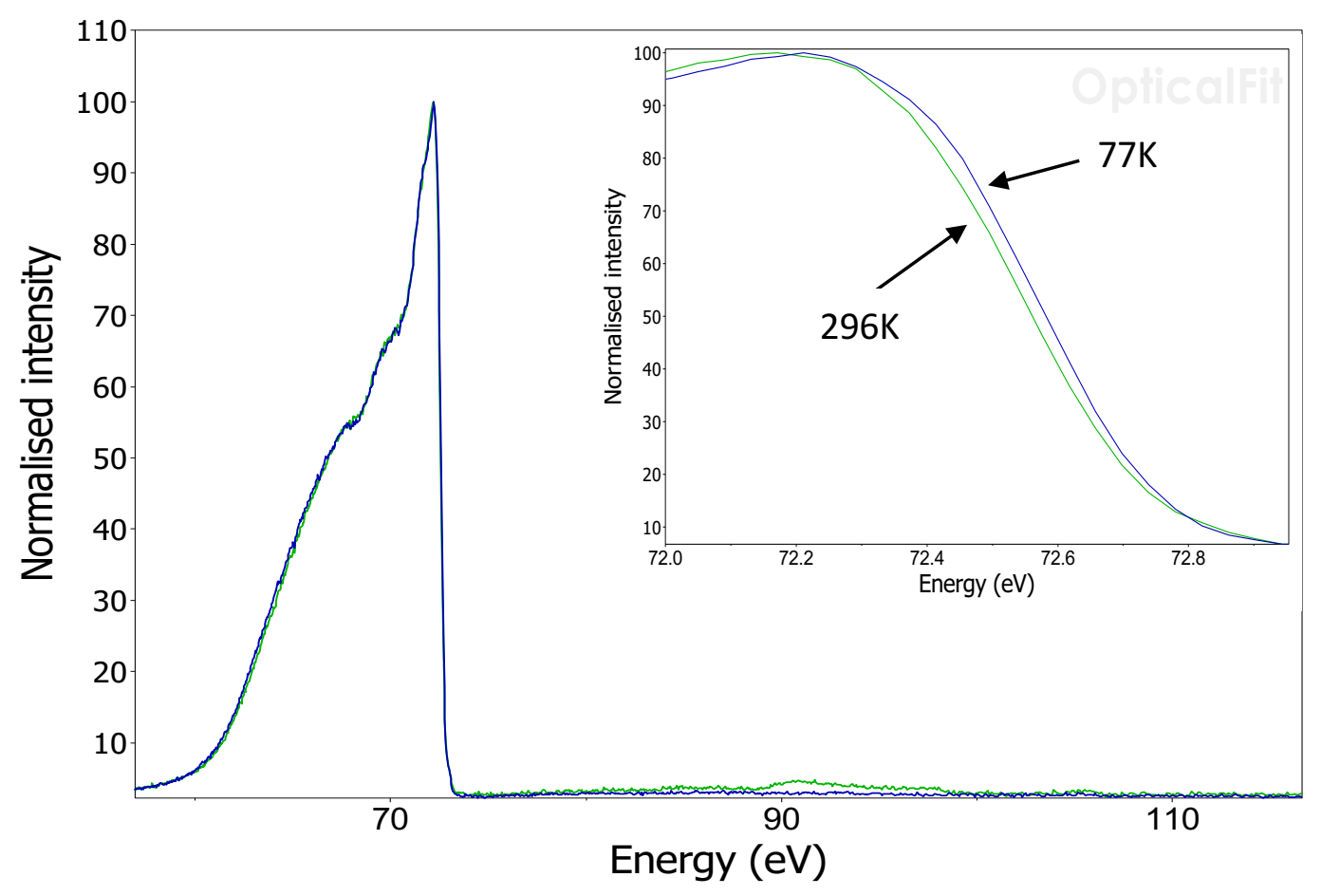

Figure 1. Al; L spectroscopy observed at room and LN temperatures showing the Fermi edge at $\sim 72.5 \mathrm{eV}$. The zoomed in region, insert, shows the Fermi edge at room temperature becoming sharper at $\mathrm{LN}$ temperature. The $25 \%$ to $75 \%$ edge resolution changes from $257 \mathrm{meV}$ at $22^{\circ} \mathrm{C}$ to $223 \mathrm{meV}$ at $\mathrm{LN}$ temperature.

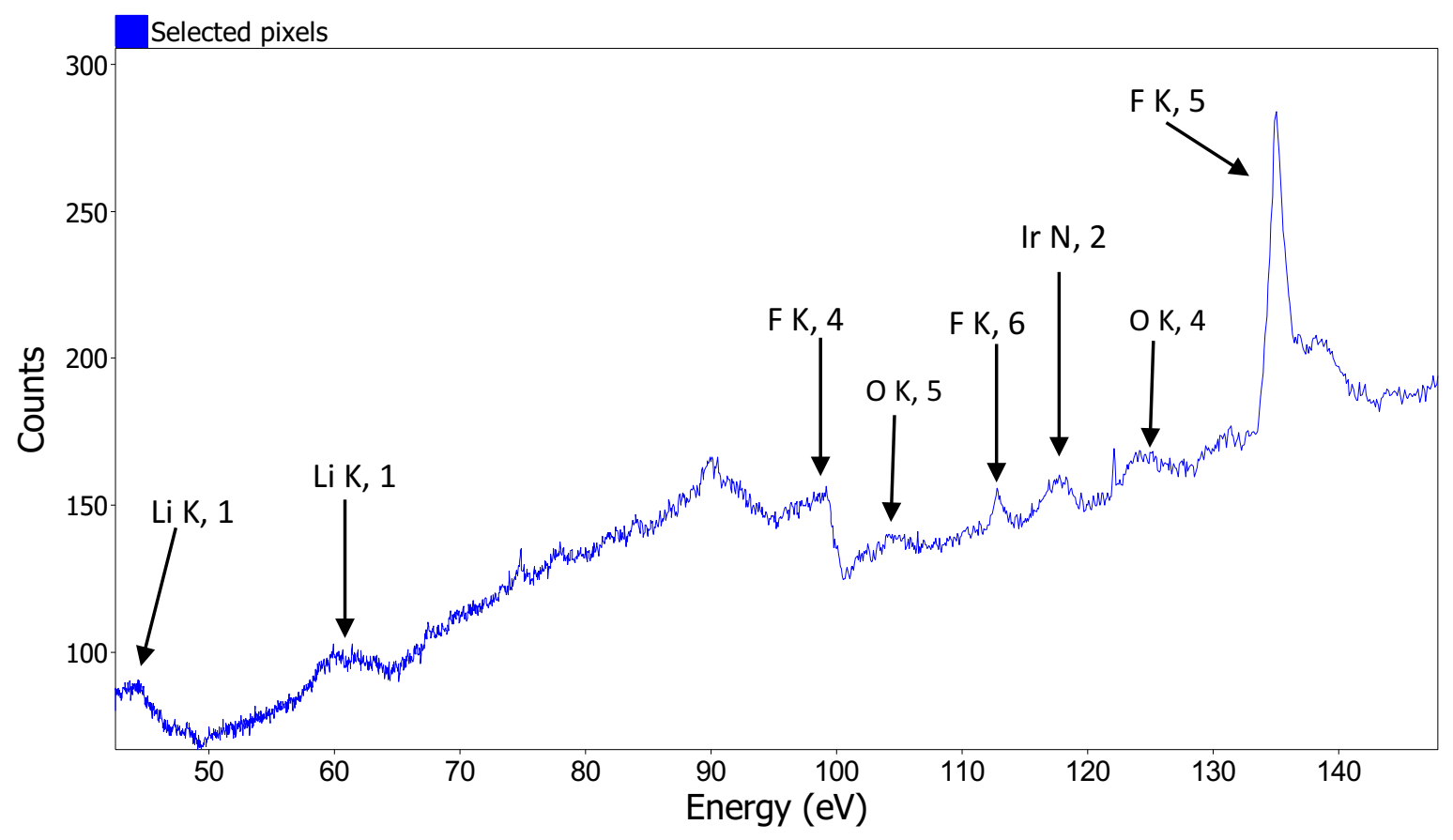

Figure 2. Spectra collected on a $\mathrm{LiF}$ showing a splitting of the $\mathrm{Li} ; \mathrm{K}$ emission. The numbers associated with each line indicate the order of the reflection. The sloping background is due to light emitted from the LiF. The $\mathrm{LiF}$ was coated with a metallic film of Ir, $2 \mathrm{~nm}$. 